\title{
EchoGéo
}

$58 \mid 2021$

Varia

\section{Enjeux de la mutation des techniques artisanales sur la formalisation de l'exploitation de l'or au Burkina Faso}

Edith Sawadogo et Dapola Evariste Constant Da†

\section{(2) OpenEdition \\ Journals}

Édition électronique

URL : https://journals.openedition.org/echogeo/22710

DOI : $10.4000 /$ echogeo.22710

ISSN : 1963-1197

Éditeur

Pôle de recherche pour l'organisation et la diffusion de l'information géographique (CNRS UMR 8586)

Référence électronique

Edith Sawadogo et Dapola Evariste Constant Dat, « Enjeux de la mutation des techniques artisanales sur la formalisation de l'exploitation de l'or au Burkina Faso », EchoGéo [En ligne], 58 | 2021, mis en ligne le 31 décembre 2021, consulté le 12 février 2022. URL : http://journals.openedition.org/echogeo/ 22710 ; DOI : https://doi.org/10.4000/echogeo.22710

Ce document a été généré automatiquement le 12 février 2022.

EchoGéo est mis à disposition selon les termes de la licence Creative Commons Attribution - Pas d'Utilisation Commerciale - Pas de Modification 4.0 International (CC BY-NC-ND) 


\title{
Enjeux de la mutation des techniques artisanales sur la formalisation de l'exploitation de l'or au Burkina Faso
}

\author{
Edith Sawadogo et Dapola Evariste Constant Da†
}

Au Professeur Dapola Evariste Constant Da qui a brusquement été arraché à notre affection le 11 décembre 2021, trouvez en ces mots ma reconnaissance et ma gratitude pour votre accompagnement durant ces années d'études.

Edith Sawadogo

\section{Introduction}

1 Le secteur minier burkinabè, comme dans la plupart des pays en développement, est caractérisé par une forte "informalité ${ }^{1}$ " de son sous-secteur artisanal. Cette informalité, qui ne contribue pas favorablement au développement économique des États, a participé pendant longtemps à la perception de l'exploitation artisanale de l'or sous l'angle de ses conséquences néfastes (Almaden, 2015). Ainsi, sans oublier ses effets positifs, elle a été largement considérée par les gouvernements et les bailleurs de fonds internationaux comme un facteur de dégradation de l'environnement (déforestation, pollution des eaux et des sols) que plusieurs études ont contribué à mettre en évidence (Bohbot, 2017 ; Bamba et al., 2013 ; Hilson, 2002). Elle est également associée au travail des enfants, à la prostitution et à la délinquance (Beavis et McWilliam, 2018 ; Richard et al., 2015 ; Human Rights Watch, 2011).

Dans ce contexte, au regard de la contribution du secteur minier industriel légal à l'économie formelle, les acteurs internationaux et nationaux, après avoir encouragé l'industrialisation, sont unanimes sur le fait que la formalisation est la solution aux nombreux problèmes sociaux et environnementaux causés par l'exploitation informelle des mines artisanales et à petite échelle. Ainsi, pour les bailleurs de fonds, toute 
stratégie de développement du secteur minier artisanal devrait mettre l'accent sur la formalisation (UNITAR et ONU Environnement, 2018; Siegel et Veiga, 2009). La formalisation est un «processus cherchant à intégrer le secteur de l'Exploitation minière artisanale et à petite échelle (EMAPE) d'or au sein de l'économie formelle de la société et du système réglementaire » (UNITAR et ONU Environnement, 2018, p. 10).

Dans le but de promouvoir la formalisation, les bailleurs de fonds internationaux comme la Banque mondiale trouvent qu'a priori celle-ci entraine l'amélioration des techniques d'exploitation minière artisanale (UNITAR et ONU Environnement, 2018). Selon cette vision, l'établissement de droits de propriété incite les promoteurs à l'investissement, d'où une amélioration technique et une augmentation des rendements dans un contexte qui protège mieux l'environnement et favorise le développement local tout en étant économiquement bénéfique pour les États. Cette vision développée depuis les années 1990 dans le cadre de l'ajustement structurel a permis la mise en œuvre de 35 programmes de réformes minières entrepris par la Banque mondiale dans 24 pays africains, entre 1988 et 2010 (Maréchal, 2013).

Dans ce cadre, des projets comme le Projet de renforcement des capacités nationales du secteur minier et de gestion de l'environnement (PRECAGEME), financé par la Banque mondiale, ont vu le jour en 1998 au Burkina Faso. Dans le but d'encadrer ce secteur, le PRECAGEME avait d'une part, encouragé une formalisation de l'activité minière artisanale à travers l'établissement des documents de propriété. Il s'agit, selon le code minier de 1997 reconduit en 2003 et 2015, d'autorisations d'exploitation artisanale de l'or, attribuées ${ }^{2}$ pour une durée de deux ans et renouvelable. C'est un outil juridique nécessaire pour entreprendre toute activité d'exploitation artisanale légale de l'or au Burkina Faso (Côte, 2013). Son imposition a cependant contribué à la marginalisation de petits exploitants miniers au profit d'une élite (Côte, 2013; Fisher, 2007). En effet, l'inadéquation des structures et des politiques à l'exploitation minière artisanale constituerait des éléments d'exclusion et de discrimination des petits exploitants miniers (Tschakert et Singha, 2007). Depuis le dernier code de 2015, d'autres documents tels que les cartes d'artisans miniers sont introduites par le gouvernement.

D'autre part, le PRECAGEME avait mis l'accent sur l'amélioration des techniques d'exploitation minière artisanale et la promotion de celle à petite échelle. Il s'agit essentiellement ici d'améliorer les techniques de l'exploitation artisanale de l'or en amenant progressivement les promoteurs vers les exploitations semi-industrielles et industrielles formelles. Dans ce cadre, des techniques d'extraction et de traitement du minerai aurifère ont été introduites dans des sites pilotes pour maximiser le taux de récupération de l'or (ministère des Mines et de l'Énergie, 2013 ; World Bank, 2005). À long terme, ces démarches promues par les bailleurs de fonds imposant d'abord l'établissement de droits de propriété artisanale ont montré leurs limites à travers la marginalisation et la discrimination des exploitations minières non formelles.

De nos jours, dans des sites aussi bien formels qu'informels, des techniques d'exploitation artisanale de l'or sont de plus en plus améliorées avec la mécanisation de l'exploration et de l'extraction de l'or alluvionnaire par des détecteurs de métaux (Bolay, 2016 ; Calkins, 2016 ; Dessertine, 2016), l'usage de moulins chinois au Soudan (ou bouffe-tout au Burkina Faso) qui broient et concentrent en même temps le minerai contenant du mercure (Sawadogo, 2021; Gagnol et al., 2019), l'usage de moyens améliorés dans le fonçage, la protection des puits et la mécanisation de la transformation du minerai filonien en poudre (Sawadogo, 2021, 2011 ; Mégret, 2013). 
Ces techniques permettent aux exploitants de franchir les obstacles physiques (dureté des roches, nappes phréatiques), mais ne sont pas sans conséquences sur l'environnement.

7 L'amélioration des techniques d'exploitation, associée à l'augmentation du nombre d'exploitants, constituerait, à l'image de précédentes stratégies de formalisation aux États-Unis (La Croix, 1992 ; Libecap, 1986 ; Umbeck, 1977), des atouts pour entamer une réelle formalisation à travers l'établissement de documents de propriété. En effet, selon ces auteurs, l'augmentation des investissements avait poussé les exploitants artisanaux à aller à la recherche de titres de propriété pour sécuriser leurs investissements.

Tout comme dans le cas californien où les investissements ont devancé l'établissement des droits de propriété, dans plusieurs sites d'orpaillage du Burkina Faso et notamment dans la commune de Kampti, des investissements ont permis l'amélioration des techniques dans des sites qui restent non formels. Il convient alors de chercher à comprendre pourquoi dans un contexte d'attractivité mondiale de l'or du fait de son prix élevé depuis la deuxième moitié des années 2000, l'amélioration des techniques d'exploitation incitée par les bailleurs de fonds n'a pas favorisé une formalisation effective. Comment les techniques d'extraction peuvent-elles contribuer à la formalisation du secteur? Quel est le niveau d'évolution des techniques d'exploitation et leurs impacts sur l'environnement? Pourquoi les sites restent dans l'informalité malgré les nouvelles techniques utilisées?

Cet article contribue au débat sur la formalisation du secteur minier artisanal de l'or à travers une recherche documentaire et des travaux de terrain réalisés dans la commune de Kampti durant les mois de mai 2018 et décembre 2019 (illustration 1). Une recherche documentaire sur les techniques d'extraction et la formalisation ainsi que notre expérience du terrain minier artisanal datant de 2009 ont permis d'établir les changements et améliorations intervenus dans le secteur en termes de techniques d'exploitation et de politiques de formalisation. Lors des travaux de terrain, nous nous sommes intéressés aux techniques utilisées dans l'extraction et le traitement de l'or ainsi qu'à leurs empreintes possibles sur les composantes de l'environnement (sols, végétation, eau). Les dépenses qui incombent à leur fonctionnement ou à leur achat ont été dans la mesure du possible abordées avec des responsables de puits. Aussi, la perception de la dégradation de l'environnement par les populations locales a été également abordée.

10 La commune de Kampti est une des neuf communes rurales de la province du Poni. Elle a vu l'arrivée de premiers orpailleurs de filons en 1998 dans le village de Maména à la même période que dans d'autres communes de la région du Sud-Ouest du Burkina Faso (Werthmann, 2003). La ruée vers l'or dans la commune n'a effectivement eu lieu qu'en 2005 après la découverte en fin 2004 d'une pépite d'or dans le terroir villageois de Fofora par des orpailleurs précédemment installés à Maména (Mégret, 2013). Depuis, plusieurs sites d'extraction artisanale de l'or ont vu le jour de façon plus ou moins éphémère. En décembre 2019, la commune comptait 6 campements miniers, dont Fofora, Maména, Galgouli, Bantara, Samgboulanti et Nielka (illustration 1) et environ 1 500 puits d'extraction (Sawadogo, 2021). 


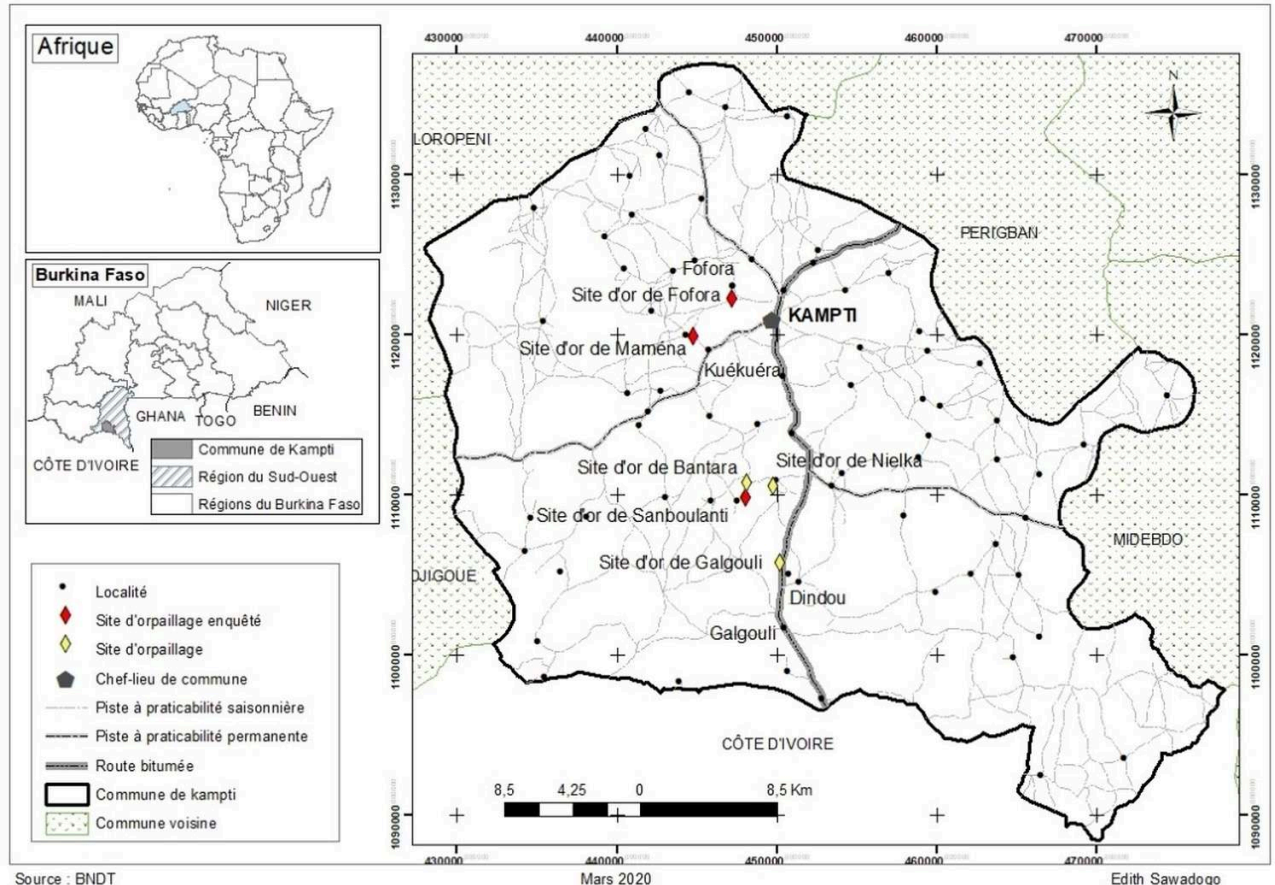

Source : BNDT. Auteur : E. Sawadogo l'or et leurs enjeux environnementaux. Dans la seconde, les acteurs et leurs rôles dans les chaînes de production, de commercialisation et dans la recherche de formalisation sont abordés. Dans la troisième partie, les attitudes des acteurs face à la formalisation de sa production et de sa commercialisation timidement entamée depuis 2018 par Agence nationale d'encadrement des exploitations minières artisanales et semimécanisées (ANEEMAS) sont analysées.

\section{Évolution des techniques d'extraction et enjeux environnementaux} physico-chimique " (Mauss cité par Coavoux, 2013, p. 2). Dans cet acte, des outils plus ou moins efficaces, progressivement améliorés, sont utilisés dans un processus bien défini pour atteindre des objectifs. Elle est le fait d'une communauté et comporte des conséquences sur l'environnement physique (Coavoux, 2013). Dans le secteur minier artisanal de l'or, l'amélioration des techniques qui est encouragée par les bailleurs de fonds dans le but de maximiser le profit à travers l'augmentation des rendements n'est pas sans conséquence sur l'environnement physique.

L'exploitation artisanale de l'or comporte plusieurs étapes dont la prospection, le fonçage ou creusage des puits de mine et le traitement du minerai (transformation 
mécanique, lavage, amalgamation avec du mercure et cyanuration). Selon l'étape, différents outils aux enjeux environnementaux divers sont utilisés.

\section{Conséquences environnementales de l'évolution des techniques de prospection et d'extraction de l'or}

Dès le $\mathrm{XV}^{\mathrm{e}}$ siècle, les populations du Burkina Faso se servaient de la présence de certaines légumineuses (Bauhinia reticulata ou tipoega en mooré) et ébénacée (Diospyros mespiliformis ou Gaaka en mooré) comme indicateurs de la présence de l'or (Kiéthéga, 1983, p. 134). Dans les années 1980, le recrutement des populations locales pour des travaux de recherches géologiques par le Bureau voltaïque de la géologie minière devenu le Bureau des Mines et de la Géologie du Burkina depuis 1984, leur a permis de se familiariser avec les méthodes modernes de prospection (Zonou, 2005). Sur la base de ces acquis, la prospection artisanale consiste en l'observation de roches et de filons de quartz pour déceler la présence de pyrite communément appelée «kiri» par les orpailleurs. Depuis 2009, des détecteurs de métaux, dont le prix allait en $2014^{3}$ de 650 000 FCFA (environ $1000 €$, pour le X-TERRA705 qui détecte les métaux à $1,5 \mathrm{~m}$ de profondeur), à 1800000 FCFA (environ $2800 €$, pour le GPX5000 qui détecte les métaux jusqu'à $8 \mathrm{~m}$ de profondeur) sont utilisés. Ces appareils, principalement utilisés dans la recherche de pépites d'or alluvionnaires, permettent aussi la découverte de nouveaux sites d'extraction de filons.

16 Cette innovation dans la prospection artisanale de l'or a des effets sur l'environnement, notamment le retournement du sol de façon plus ou moins superficielle. Selon nos entretiens, l'impact de ces détecteurs est beaucoup plus craint et dénoncé par la population locale que le creusage des puits filoniens localisés dans certains cas sur de faibles superficies et sur des terres non cultivées. Cependant, les exploitants artisanaux d'or, essentiellement allochtones venus d'autres régions du Burkina Faso (Sawadogo, 2021; Gros et Mégret, 2018 ; di Balme et Lanzano, 2013, 2014), prennent moins en considération les conséquences néfastes du détecteur. Selon eux, d'une part, l'usage du détecteur sur certaines terres non cultivées serait bénéfique à l'exploitation agricole. Parce que la dégradation n'est pas définitive, surtout pour les sols retournés à $1,5 \mathrm{~m}$ de profondeur et qui sont recouverts de végétation après les premières pluies (Sawadogo, 2021). D'autre part, les sols rocailleux auparavant non exploités pour l'agriculture du fait de la difficulté du labour, sont directement semés après exploitation aux détecteurs. Nos observations sur le terrain montrent effectivement la mise en culture des terres après exploitation par ces détecteurs.

Le fonçage des puits, toujours manuel, est fait avec des pioches et des pelles servant respectivement à creuser et déblayer le minerai. Des marteaux et des burins sont utilisés pour concasser les roches dures. En fonction de l'évolution du creusage, des cordes et des sacs sont utilisés pour faire remonter le minerai (Sawadogo, 2011).

La pratique actuelle est que dans certains puits, la remontée des sacs et des exploitants est faite à l'aide de poulies fonctionnant par la force humaine ou mécanique (illustration 2 gauche). Le minerai est excavé et abandonné sur place, couvrant ainsi les arbustes, les herbes et le sol arable illustration 2 droite). 


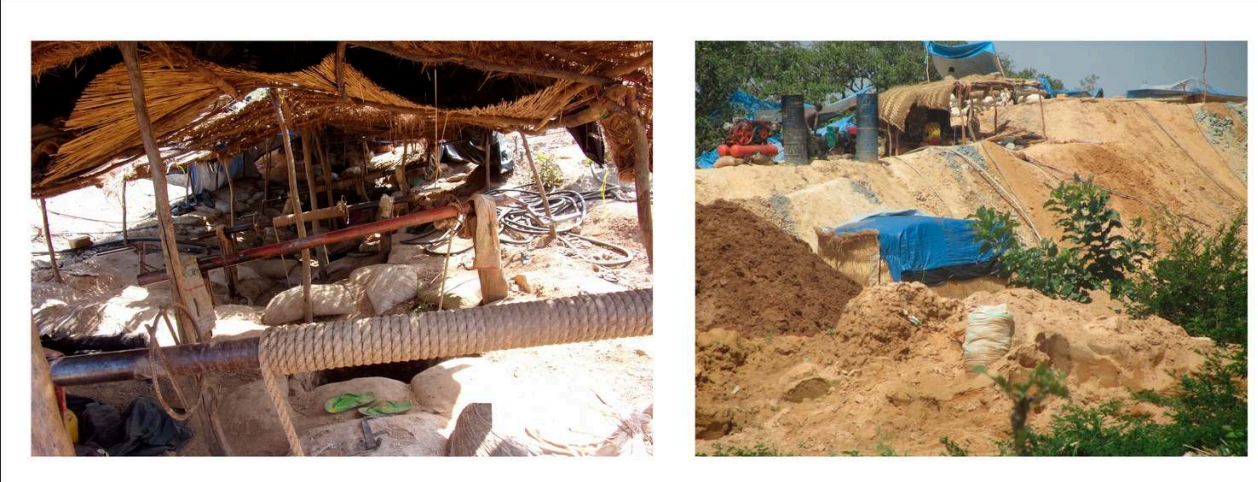

Gauche : puits d'orpaillage munis de poulies manuelles à Fofora.

Droite : site d'extraction filonien à Sangboulanti.

Auteur : E. Sawadogo, décembre 2019 et mai 2018.

19 La situation de la commune de Kampti dans le domaine soudanien où les pluies sont plus abondantes que dans les autres domaines (sahélien et soudano-sahélien) est un facteur qui fragilise les sols. Cela expose les parois des galeries et des puits à des effondrements, des éboulements et d'autres risques. Des troncs d'arbres sont alors utilisés pour renforcer la résistance des parois à travers le boisage, une technique utilisée depuis l'antiquité dans les mines d'or gauloises du sud-ouest du Massif central (Caüuet, 2000).

Le boisage des puits a cependant l'inconvénient de laisser une empreinte au couvert végétal à travers la coupe abusive du bois. Cette coupe était effectuée dans la clandestinité au début de la ruée vers l'or dans la commune en 2005. Par la suite, une entente entre propriétaires fonciers et orpailleurs a intensifié la coupe du bois avant que des alternatives à la coupe du bois ait été trouvée par les orpailleurs.

21 Une étude diachronique de la végétation de la commune de Kampti entre 2001 et 2018 montre une dégradation de la savane arbustive et une augmentation des sols nus. En effet, d'une superficie de 43128 ha en $2001^{4}$, la savane arbustive n'en occupait que 24 652 ha en 2018 (Sawadogo, 2021). Cela correspond à une superficie perdue de 18476 ha au profit d'autres types d'unités d'occupation des terres comme les sols nus (44,6\%) et les zones agricoles $(26,76 \%)$. La superficie occupée par les sols nus est passée de 1149 à 1805 ha soit une différence de 656 ha. Il s'agit d'une dégradation annuelle de 38,5 ha de 2001 à 2018. Cependant, les causes de la dynamique de ces unités sont aussi bien le fait de l'orpaillage (coupe $\mathrm{du}$ bois pour le boisage, retournement des sols), de l'augmentation des superficies agricoles que de l'augmentation des besoins de la population en bois-énergie. Ils participent à la déforestation et la dégradation du couvert végétal à travers la création d'une demande importante de bois pour le soutènement des puits et galeries, la construction des hangars et des habitats dans les camps d'orpailleurs (Bamba et al., 2013 ; ONUDI, 2009).

Pour lutter contre la dégradation du couvert végétal conformément au code de l'environnement de 2013, les orpailleurs se sont retournés vers d'autres moyens de protection des puits tels que l'importation de bois de soutènement et le bétonnage.

Ils importent de plus en plus du bois d'eucalyptus des autres régions du pays et du bois de teck de la Côte d'Ivoire (illustration 3 gauche). Il s'agit de bois de grande taille dont 
le prix unitaire varie entre 3000 et 3500 FCFA (autour de $5 €$ ). Une grande quantité de bois est nécessaire pour le soutènement d'un seul puits. La seconde alternative est le bétonnage des puits avec des agrégats et du ciment (illustration 3 droite). Le coffrage des puits est fait avec un anneau métallique de fabrication artisanale. C'est une technique empruntée aux mines semi-industrielles. Le coffrage se fait du fond vers la surface des puits et est plus coûteux que le boisage. Pour ce puits de l'illustration 3 (droite), des dépenses de plus de 10 millions de FCFA (plus de $15000 €$ ) auraient été faites aussi bien pour le fonctionnement des activités que pour le bétonnage.

\section{Illustration 3 - Bois de coffrage}
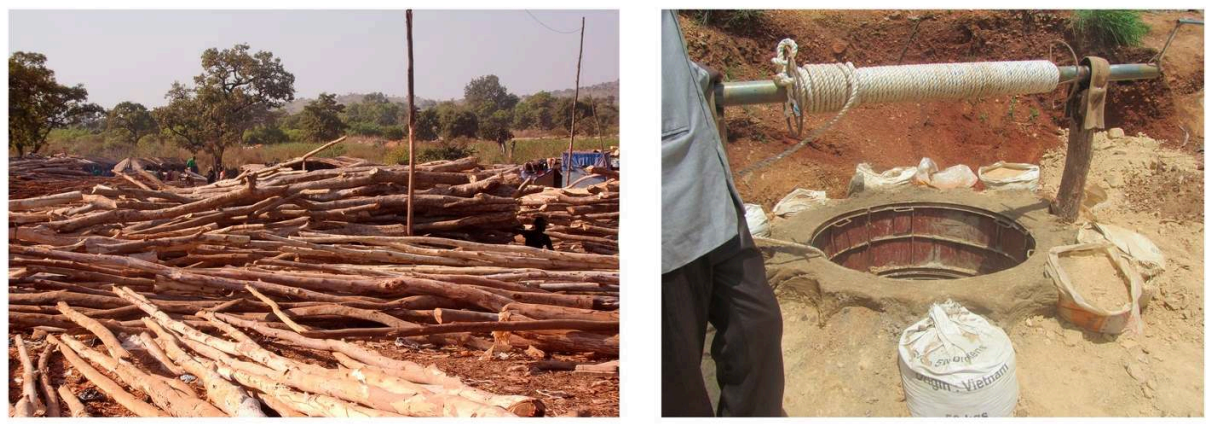

Gauche : Aire de vente de bois de soutènement de Sangboulanti.

Droite : Coffrage en béton d'un puits d'orpaillage dans le site de Maména.

Auteur : E. Sawadogo, novembre 2019 et mai 2018.

Cette technique a l'avantage d'épargner les arbres de la commune. Toutefois, dans des sites qui évoluent hors du circuit légal et formel, le risque d'abandon des puits en l'état est très élevé. Cela constituerait alors, autant que les puits, boisés ou non, des risques pour les populations et les animaux qui pourraient y chuter. Aussi, l'usage de matériaux durables comme le ciment entraverait-il toute colonisation végétale éventuelle. Après abandon, dès la première saison pluvieuse, une colonisation par des herbacées est observable ainsi qu'une remontée de la nappe phréatique favorable à la biodégradation des bois de soutènement.

\section{Les puits miniers artisanaux de l'or : de l'abandon à l'exhaure}

Pour atteindre le filon aurifère à des dizaines de mètres de profondeur, les orpailleurs utilisent une pompe d'exhaure ou un puisage afin d'évacuer l'eau issue d'une exsurgence ou de la nappe phréatique sous le site concerné. 
Illustration 4 - Motopompes utilisées
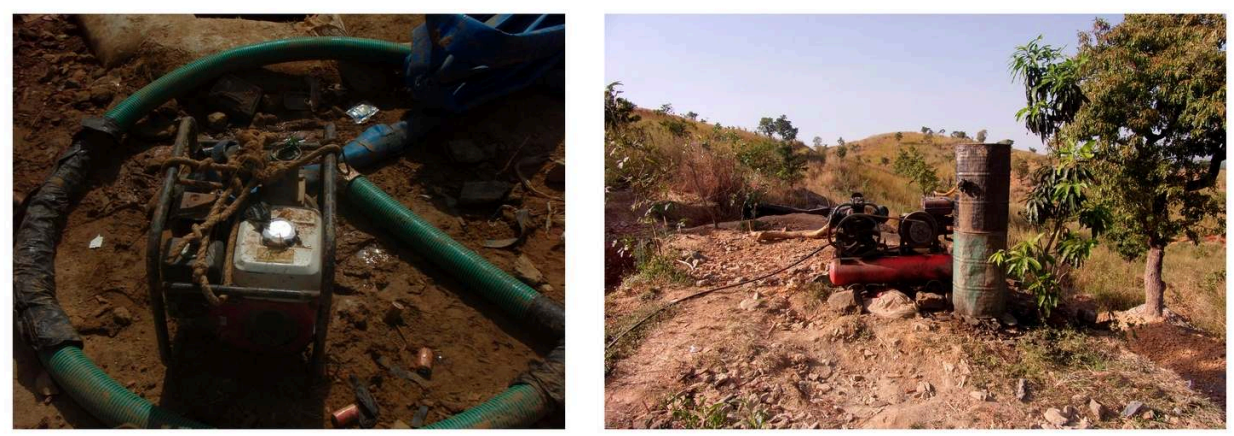

Gauche : Motopompe à réservoir incorporé, capacité environ 5 litres.

Droite : Motopompe à réservoir non incorporé à grande capacité à Fofora.

Auteur : E. Sawadogo, 2011 et novembre 2019.

26

L'exhaure correspond à cette évacuation des eaux de puits d'exploitation artisanale ou industrielle, on parle d'eaux d'exhaure. Si durant plusieurs années les puits d'orpaillage n'excédaient pas $5 \mathrm{~m}$ du fait de la présence des nappes phréatiques et de l'absence de techniques adaptées, depuis 2005, des outils de plus en plus adaptés, importés de Chine et d'Inde à moindre coût sont utilisés (Magrin, 2017).

Il s'agit de motopompes qui peuvent avoir des réservoirs à carburant incorporé. Elles sont utilisées pour pomper l'eau à l'aide de tuyaux directement plongés dans la nappe. Pour le premier type utilisé depuis le début de l'année 2000, le réservoir est incorporé à l'appareil et a de faibles capacités (illustration 4 gauche). Pour le second, de plus grande capacité, le réservoir à carburant, dissocié de la machine, est de fabrication locale. Elle peut fonctionner durant des jours sans arrêt (illustration 4 droite). Ce fonctionnement continu implique l'évacuation de milliers de mètres cubes d'eaux dans la nature, inondant certaines terres et pistes rurales comme dans le site de Sangboulanti (illustration 5). 
Illustration 5 - Site de Sangboulanti
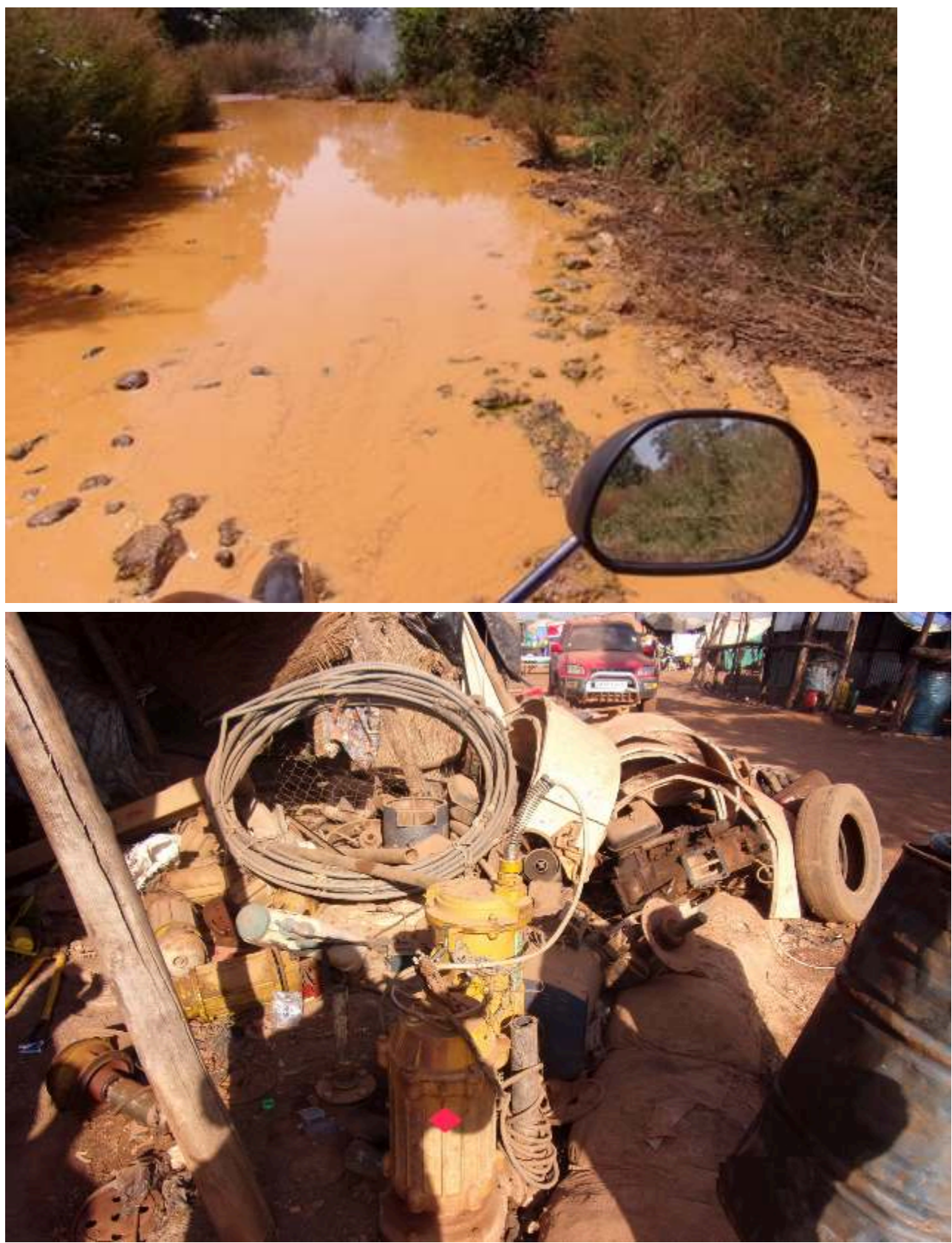

Gauche : Piste rurale inondée par les eaux d'exhaure du site d'orpaillage de Sangboulanti. Droite : Dynamo de groupe électrogène défectueux à Sangboulanti.

Auteur : E. Sawadogo, novembre 2019.

La consommation journalière en gasoil de ces appareils de grande capacité est de 2001 en 24 heures sans arrêt, soit un total de 400 litres de carburant. Cela correspondait à une consommation de $206800 \mathrm{FCFA} /$ jour (environ $300 €$ /jour) selon le prix du gasoil au Burkina Faso en 2019. Le dispositif d'exhaure à grande capacité fonctionne à l'aide de la dynamo dont la pièce de rechange peut atteindre 14 millions de FCFA (environ 21 $400 €$ ), selon un responsable du site de Sangboulanti (illustration 5 droite).

En plus de cette inondation, l'usage anarchique de carburant (gasoil) dans les motopompes expose les sites à la pollution du fait de fuites éventuelles. 


\section{techniques ainsi que l'évacuation des eaux nécessitent des coûts élevés difficilement supportables par un seul orpailleur. Cela les amène à s'associer à plus d'acteurs externes aux sites, différents des représentants des comptoirs privés d'achat et installés dans la région ou dans les grandes villes du pays. \\ Des techniques de traitement de l'or aux multiples enjeux environnementaux}

30 Le traitement de l'or commence avec un concassage du minerai extrait des puits. Dans le cadre du PRECAGEME, des concasseurs à mâchoires et des broyeurs à marteaux fabriqués localement ont été introduits dans trois sites pilotes pour maximiser le taux de récupération de l'or (ministère des Mines et de l'Énergie, 2013 ; World Bank, 2005). Des artisans locaux ont été formés à leur utilisation.

31 Ces techniques ont été vulgarisées sur toute l'étendue du territoire, favorisant la fixation des acteurs en charge de leur fonctionnement dans des campements miniers permanents.

Le minerai moulu, après concentration, subit deux types de traitement chimique: l'amalgamation au mercure et la cyanuration. L'amalgamation consiste à appliquer le mercure dans une petite quantité de minerai concentré. Elle permet de récupérer l'or sur place après le chauffage de l'amalgame or-mercure. L'or récupéré peut être vendu sur place à des patrons/acheteurs locaux ou externes au site.

Utilisé pour récupérer l'or contenu dans les rejets de lavage, le cyanure, introduit depuis 2009 dans la commune de Kampti (Sawadogo, 2011), a entrainé la recomposition des acteurs qui luttent pour posséder les rejets de traitement qui autrefois étaient abandonnés (Lanzano et di Balme, 2021). Ce processus est plus long et nécessite l'usage successif de cyanure, de zinc pour capter l'or dilué par le cyanure, de l'acide sulfurique pour soustraire l'or du zinc et enfin une attaque d'acide nitrique pour purifier l'or de l'acide sulfurique et des autres résidus (fer). La solution obtenue est raffinée dans des centres à Gaoua ou à Ouagadougou. À l'inverse de la convention de Minamata, adoptée en 2013, qui dénonce l'impact négatif et durable du mercure, la population locale a beaucoup plus peur de la pollution engendrée par la cyanuration que celle causée par l'usage du mercure. Cela s'explique par le fait que le cyanure occupe plus d'espace et en cas de ruissellement, sa consommation par le bétail peut être directement fatale. Des cas de décimation de troupeaux ont d'ailleurs été enregistrés dans la commune en $2016^{5}$. Contrairement au cyanure, le caractère bio-accumulateur du mercure ne permet pas aux populations de percevoir tout de suite ses impacts.

En dépit de la dégradation de l'environnement physique causée par les rejets de lavage du minerai aurifère, des analyses d'échantillons de sol et d'eaux dans la commune de Kampti ont souvent révélé des concentrations contraires en ce qui concerne le mercure et le cyanure. En effet, des analyses chimiques d'échantillons de sols prélevés dans le site de Fofora en 2009 montraient des terres exemptes de pollution en mercure du fait de leur faible concentration selon les normes de l'OMS 6 (Sawadogo, 2011). Cela peut s'expliquer par un usage récent du mercure qui ne date que de 2005 à Fofora. Des résultats contraires ont été obtenus en 2014 par Roamba (2014), à Galgouli, un site de la même commune, fonctionnelle depuis 2008. Au Burkina Faso, 25 tonnes de mercure seraient utilisées chaque année pour l'exploitation minière artisanale et à petite échelle

EchoGéo, 58 | 2021 
(Lassen et al., 2016). Cependant, la pollution qui en résulte n'est pas homogène d'un site à l'autre et dépend du nombre d'années d'utilisation. Elle dépend aussi de facteurs propres au site comme la richesse du substratum en mercure (Grasmick et al., 1998) ou l'intensité des activités (Beavis et McWilliam, 2018).

La contamination des sols par le cyanure est variable selon les sites, les saisons, mais également selon la proximité avec le lieu de traitement du minerai. Des études d'échantillons d'eau et de sol dans le bassin versant de Galgouli ont montré des concentrations en cyanure pouvant dépasser jusqu'à quarante fois la valeur guide de l'Organisation mondiale de la santé (OMS) de la teneur en cyanure et cyanure total (Razanamahandry, 2017). Cependant, selon ses résultats, la concentration décroit du point de cyanuration vers l'exutoire et de la saison sèche à la saison pluvieuse.

Dans cette brève explication des conséquences des techniques d'extraction et de traitement du minerai, la mécanisation reste faible. Les techniques sont plus mécanisées dans l'extraction et le traitement mécanique (concassage et mouture) que pour le traitement chimique. Des coûts importants sont mobilisés pour l'achat du matériel et pour son fonctionnement continu. L'amélioration des techniques d'extraction et de traitement promue depuis plusieurs décennies par la Banque mondiale à travers le monde a permis non seulement une augmentation des rendements, mais aussi celle des conséquences sur l'environnement. Cependant, ces sites restent dans l'informalité.

\section{Rôles des exploitants dans la chaîne de production et de commercialisation : obstacles à la recherche de formalisation}

Les techniques d'exploitation de l'or restent faiblement mécanisées, mais mobilisent d'importants investissements et coûts de production.

\section{Acteurs de l'extraction de l'or : rôles dans la quête de la formalisation}

Dans les sites, les exploitants sont organisés dans l'exécution de leurs activités (Lanzano et di Balme, 2014). De la prospection à la récupération de l'or, les artisans sont organisés de sorte que sur toute la chaîne, les acteurs qui interviennent perçoivent une part de la rente aurifère. Si les creuseurs et le propriétaire de trou se partagent le minerai extrait ( $50 \%$ pour le chef de trou et le reste pour les creuseurs), le concasseur prend 1000 FCFA (moins de $2 €$ ) par sac de $50 \mathrm{~kg}$, les propriétaires de hangar, des femmes principalement, fournissent l'eau de lavage aux propriétaires du minerai et gardent le minerai issu de la concentration encore appelée boue selon Lanzano et di Balme (2021). Les résidus de lavage, les haldes, gardés par le propriétaire de hangar, sont vendus après plusieurs stockages à des millions de FCFA à des fins de cyanuration. Selon des détenteurs de hangar, un chargement de véhicule benne 10 roues serait vendu à plus ou moins 1 million de FCFA (environ $1500 €$ ) selon la négociation et la richesse de la boue. Malgré ces coûts, ce retraitement semble lucratif dans la mesure où le mercure ne permet de récupérer que $40 \%$ de l'or contenu dans le minerai (Gagnol et al., 2019). Au-delà du travail par affinité, les exploitants de minerai travaillent en 
équipe sous la responsabilité d'un chef de puits qui fait fonctionner les travaux à l'aide de financements de patrons.

Pour faciliter la relation avec les autochtones, des redevances sont collectées auprès des propriétaires de hangars de traitement, de moulins, des commerces par les responsables de comptoir et versés en partie aux propriétaires terriens. Dans le but de résoudre des problèmes financiers ponctuels, les responsables d'un site peuvent procéder à des cotisations auprès des détenteurs de puits et de commerce du campement minier. En mai 2020, les orpailleurs ayant des puits à Sangboulanti ont procédé à une cotisation pour le remblaiement des puits abandonnés. Cette cotisation, à hauteur de 20 millions de FCFA (environ $3500 €$ ), centralisée auprès de représentants de comptoirs installés à Bantara, est la preuve que la formalisation peut être possible en se basant sur l'organisation interne des sites et en imposant aux comptoirs d'achat l'acquisition d'autorisation d'exploitation artisanale ou semi-mécanisée de l'or. Des coopératives d'exploitants miniers artisanaux ou des intermédiaires peuvent sur cette base être créées comme le recommandent le code minier de 2015 et la Banque mondiale (UNITAR et ONU Environnement, 2018). Cela pourrait cependant contribuer à recomposer une fois de plus la hiérarchie locale de l'orpaillage aux dépens de certains acteurs émergents depuis 2014.

Depuis 2014, on constate une diminution de pouvoir des comptoirs privés d'achat suite à l'insurrection populaire. En effet, l'année 2014 a été marquée au Burkina Faso par l'insurrection populaire, fin octobre ${ }^{7}$, suite à laquelle les représentants de comptoirs privés d'achat qui contrôlaient l'organisation et le financement de l'exploitation artisanale de l'or (di Balme et Lanzano, 2014) avaient fugué. Cela a favorisé l'implication, dans le financement des travaux, de nouveaux acteurs externes aux sites. Les patrons externes sont actuellement des acteurs clés de l'exploitation aux côtés des comptoirs privés d'achat à nouveau présents et qui restent au centre de la gouvernance (Lanzano et di Balme, 2021). Leur présence constitue une base pour aller vers la formalisation.

41 En s'appuyant sur les comptoirs d'achat déjà présents, il est possible d'associer les différents patrons à travers une approche intégrée des différents acteurs de l'orpaillage, même si forcément certains devraient se reconvertir ou disparaître selon la nouvelle organisation de l'ANEEMAS. Des compromis seront nécessaires aussi bien du côté des patrons que des comptoirs privés qui, jusqu'à présent, sur la base de leurs agréments d'achat, organisaient et contrôlaient les activités dans les sites d'orpaillage de la commune. Cela, sans être responsables des impacts environnementaux engendrés.

La mécanisation de l'exploitation artisanale de l'or associée à l'organisation interne des sites a stimulé l'envie de formaliser les activités dans le but de sécuriser les investissements. Selon les responsables d'un des sites visités, des démarches ont été entreprises pour l'obtention d'une autorisation d'exploitation artisanale de l'or sans succès. En effet, ces acteurs se sont heurtés à des obstacles d'ordre juridiques.

L'informalité des sites visités n'est pas ainsi due à la passivité des acteurs, mais à plusieurs facteurs, dont la priorisation des exploitations à grande échelle au mépris des petits exploitants qui se voient refuser les autorisations d'exploitation artisanale de l'or sur des périmètres d'exploration ou des permis industriels (Geenen, 2012).

Selon les codes miniers de 1997 et 2003 du Burkina Faso, le renouvellement d'une autorisation d'exploitation artisanale d'or était impossible en cas d'attribution de titre 
minier à grande échelle sur une partie ou toute la superficie occupée par l'autorisation (articles 38, 46, 73 des codes miniers respectifs de 1997, 2003 et 2015). Ces conditions, qui paraissaient rudes dans les textes, présentaient une certaine souplesse du fait de l'absence d'une précision à propos de l'obtention de l'accord du détenteur du permis industriel s'il y a lieu. Dans les faits, les acteurs obtenaient des autorisations pour avoir été les premiers à demander des titres de propriété. Depuis le code de 2015, il est difficile de superposer des titres miniers à grande échelle et des autorisations d'exploitation artisanale. En effet, une des conditions d'obtention de l'autorisation d'exploitation minière artisanale de l'or est l'accord écrit du détenteur de titre minier, qui leur est très souvent refusé. Cela facilite l'exploitation illégale de l'or par les détenteurs d'agréments d'achat que nous observons d'une manière générale dans les sites visités. Cette situation est similaire au cas guinéen où des détenteurs de permis d'exploration exploitent l'or de façon formelle ou informelle. Il en résulte ainsi une discrimination des petits artisanaux qui se retrouvent dans des sites sans autorisation légale comme le cas du site de Maména, selon un des responsables.

En dépit des contraintes juridiques qui ne favorisent pas la formalisation effective, la nouvelle agence ANEEMAS cherche à prendre le contrôle de ces sites qui fonctionnent sans autorisation d'exploitation artisanale de l'or au titre de l'article 26 de la Loi 028-2017 portant organisation de la commercialisation de l'or et des autres substances précieuses au Burkina Faso. S'opposant ainsi à des acteurs à qui des autorisations d'exploitations artisanales ont été refusées.

\section{Organisation de la commercialisation et possibilité de formalisation}

En fonction de la source de financement, l'or obtenu par les exploitants est vendu en partie aux patrons et aux comptoirs privés d'achat présents sur les sites. Dans le circuit de production, chaque acteur est un éventuel commerçant (Alvarez et al., 2016) et les prix varient selon la chaîne de commercialisation. Le patron fixe le prix d'achat de l'or en deçà du prix sur le site et sur le marché international. Il est chargé ensuite de la vente aux acheteurs régionaux, nationaux et/ou internationaux. Il existe plusieurs patrons indépendants, contrairement au cas de la République Démocratique du Congo (RDC). Selon Geenen, dans les sites d'exploitation artisanale de l'or de Kalanga et Lukwera, les exploitants s'associent à des acteurs qu'ils ont antérieurement rejetés, pour empêcher l'installation de nouveaux acteurs (Geenen, 2014). En Mauritanie, les patrons sont également des commerçants ou des exportateurs d'or (Gagnol et al., 2019). Dans la commune de Kampti, les exploitants sont tenus par une obligation morale et de dette pour traiter avec leur patron. Cependant, il peut y arriver que le puits exploité ne soit pas productif. Une dette demeure alors entre patrons et exploitants. Même en cas d'association avec un nouveau financier, l'exploitant reste redevable à son précédent créancier.

47 L'arrivée effective de l'ANEEMAS en 2018 après avoir été créée par le décret $n^{\circ}$ 2015-1420/ PRESTRANS/PMMEF/MME du 30 novembre 2015, constitue un facteur de bouleversement de toute la chaîne de commercialisation (illustration 6). 


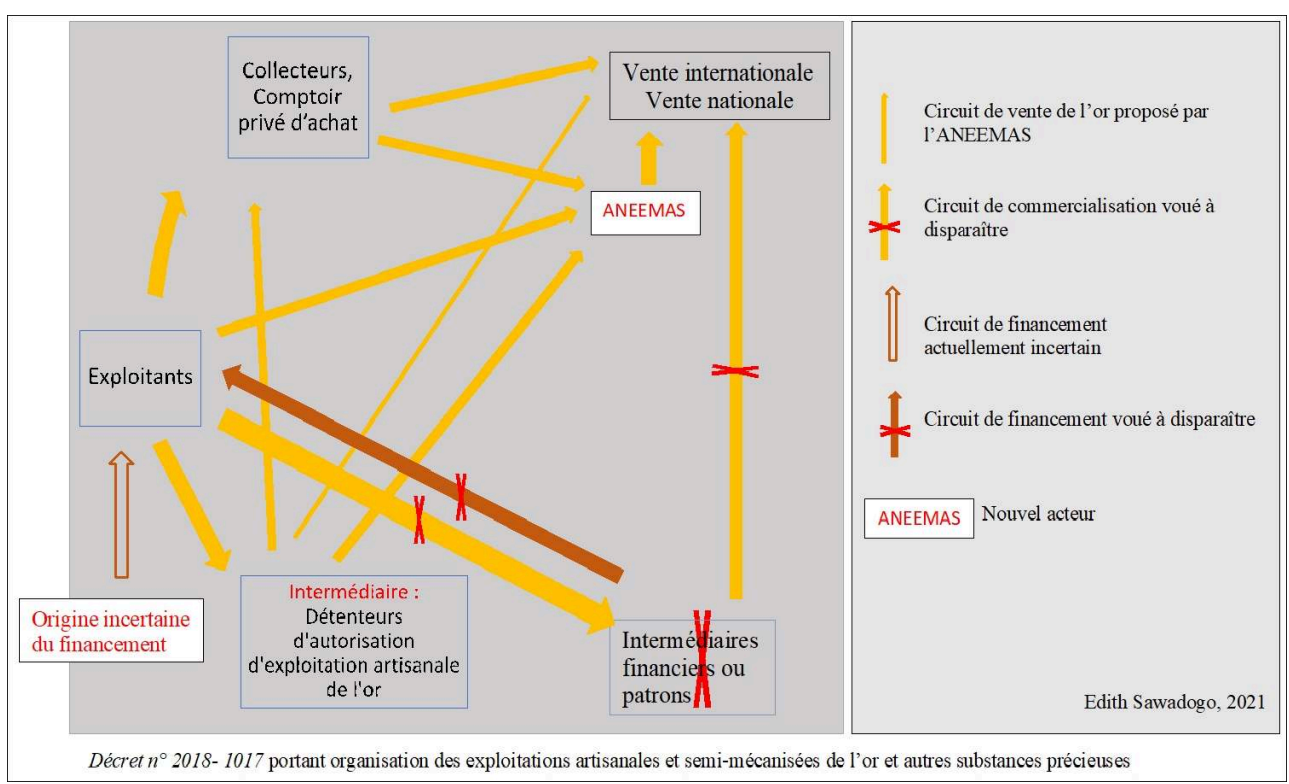

Auteur: E. Sawadogo, 2021.

L'importance de la fraude dans le secteur minier artisanal informel a contribué à la tenue de discours discriminatoires et marginalisant les nouveaux patrons intermédiaires financiers. Le discours dominant les indexe comme les seuls à tirer profit de l'orpaillage au détriment des populations locales et des petits exploitants. Leur suppression, à travers la formalisation, est alors perçue comme un moyen de mieux canaliser les recettes du commerce de l'or par quelques acteurs dont les gouvernants auront la maîtrise. La diminution du nombre d'acteurs finançant les activités pourrait-elle permettre à l'État de contrôler le commerce de l'or ? Cela n'est pas évident, car la commercialisation de l'or est disséminée au sein des communautés minières d'où la difficulté du contrôle par l'État (Alvarez et al., 2016). La mise à l'écart de ces acteurs pourrait tout autant favoriser l'intensification de la fraude et l'illégalité. Ailleurs, comme en Colombie, l'expérience a montré que la mise à l'écart de certains acteurs hors du circuit de formalisation ne faisait que renforcer l'illégalité de l'activité et donc la fraude (Veiga et Marshall, 2018). En effet, les nouveaux textes mettent en scène les exploitants qui devraient chercher d'autres sources de financement, différentes de l'ancienne (illustration 6). L'or obtenu doit être vendu à des collecteurs, à des intermédiaires détenteurs de conventions de gestion, aux détenteurs d'autorisations d'exploitation artisanale ou à l'ANEEMAS. Ces différents acteurs qui ne font peut-être pas partie du circuit de financement constituent des échelles de vente où le prix diffère d'un acteur à l'autre.

Nous savons que l'obtention de crédit dans les institutions financières est une difficulté majeure dans ce secteur. L'association des patrons intermédiaires financiers dans la quête de la formalisation a déjà démontré ces avantages aux Philippines, précisément à T'boli. Dans le processus de formalisation, les patrons constituent le pilier et sont chargés d'identifier des exploitants dans le circuit légal (Verbrugge et Besmanos, 2016). Dans le cas de la commune de Kampti, des rencontres de l'ANEEMAS avec les patrons pourraient aussi bien faciliter l'identification des acteurs avec qui ils travaillent. L'élimination de certains intermédiaires financiers par l'ANEEMAS est à l'origine du 
mécontentement des orpailleurs face à la formalisation, envisagée à travers des autorisations d'exploitation artisanale de l'or et de la délivrance de cartes d'artisans miniers.

\section{Attitudes des acteurs de l'orpaillage vis-à-vis des tentatives de formalisation}

50 Dans le but de formaliser l'orpaillage, l'ANEEMAS tente d'organiser les exploitants à travers l'établissement d'autorisations d'exploitation artisanale pour des personnes physiques, des coopératives ou des intermédiaires qui vont travailler dans certaines conditions pour l'agence ${ }^{8}$. Des cartes d'artisans miniers sont aussi en cours d'élaboration pour les différents acteurs.

51 Depuis la volonté affichée de l'État de formaliser à nouveau le secteur avec le début des activités de l'ANEEMAS en 2018, les orpailleurs ont des attitudes mitigées, voire réfractaires. Cela s'explique par l'enrichissement de nouveaux patrons qui financent les activités depuis 2014, avec le départ momentané des responsables de comptoirs privé d'achat. Pour ces acteurs devenus économiquement puissants, l'encadrement par l'État est perçu comme un retour à la situation de pauvreté passée. L'organisation des sites par les comptoirs privés d'achat, qui prévalait avant 2014 (di Balme et Lanzano, 2014) a été bouleversée avec l'insurrection. Les responsables des différents sites présentaient une résistance à l'encadrement lors de nos entretiens en décembre 2019. Cependant, visiblement, la crainte d'une éventuelle fermeture des sites demeure. En effet, après une longue période de fermeture du site de Fofora de novembre 2017 à mars 2019 par une décision du Gouverneur de la région, une autre fermeture serait de trop. Dans ce secteur où les acteurs travaillent majoritairement hors des circuits formels, les menaces d'expulsion sont utilisées par des gouvernants comme des outils de pression. L'expulsion intervient souvent en réponse à l'application des textes juridiques en matière de formalisation qui marginalise les artisans miniers au profit des sociétés industrielles ou semi-industrielles formelles (Bolay, 2018). Dans d'autres cas, comme à Fofora en 2017, des conflits entre orpailleurs allochtones et populations autochtones avaient amené l'administration à faire valoir son monopole de violence à travers l'expulsion (Sawadogo et Da, 2020 ; Gros et Mégret, 2018).

L'opposition des exploitants sur le terrain constitue un frein à l'avancée des activités de l'agence. En effet, dans le cadre de la mise en œuvre du projet « Appui à l'encadrement du secteur aurifère artisanal " prévu pour durer de 2019 à 2021, les activités de formalisation dans le site pilote de Fofora n'avaient pas encore démarré en décembre 2020. Même le matériel mécanique de traitement du minerai n'avait pas été livré, selon un responsable orpailleur de la commune. En dépit des contraintes sur le terrain, une orientation politique de l'agence pourrait expliquer ces retards. L'accent semble être essentiellement mis sur la formation d'agents affectés dans différentes régions du pays. En 2020, seulement dix bureaux déconcentrés, composés de deux agents par Bureau, existaient sur toute l'étendue du territoire. L'insuffisance de l'effectif des encadrants causait problème.

53 La concrétisation de l'appui technique de l'État pourrait inciter les acteurs à la formalisation. Selon les orpailleurs interviewés, l'approche de l'agence pour l'établissement des documents de propriété est mauvaise. Au lieu de proposer d'abord des sources de financement, elle impose la manière dont elle achète l'or que les 
orpailleurs ont extrait à l'aide de crédits obtenus auprès de leurs patrons. L'appui technique par l'État a fait ses preuves dans d'autres pays comme le Soudan, où l'État a pu relativement contrôler la production et la vente de l'or à travers l'établissement de droits de propriété (Chevrillon-Guibert, 2017). Même si l'encadrement du commerce de l'or a montré plus tard ses limites dans ce pays (Chevrillon-Guibert et Magrin, 2018), l'État a fait un effort important, principalement par la centralisation du traitement et l'octroi de matériel de traitement. Ce qui lui a permis d'augmenter ses recettes de l'exploitation artisanale de l'or.

Des initiatives privées sont développées dans le sens de la mécanisation du traitement et de la récupération de l'or dans trois communes du Ganzourgou (Boudry, Méguet, Zorgho) par l'Alliance pour une mine responsable (ARM) (Medinilla et al., 2020). L'ARM est un acteur clé dans ce domaine. Il a introduit des concentrateurs centrifuges dans ces communes. Ces techniques étant coûteuses et nécessitant une maîtrise importante $\mathrm{du}$ fonctionnement des appareils, les exploitants artisanaux des sites de notre étude s'opposent, pour le moment, à toute mesure de formalisation (titre de propriété des sites d'extraction et de traitement, carte d'artisan minier).

En plus de la proposition de prix d'achat (Medinilla et al., 2020), la mise à l'écart des patrons semble aussi constituer un frein à la formalisation des activités d'extraction et de traitement, mais aussi de la commercialisation de l'or.

Bien que l'encadrement proposé par l'ANEEMAS passe aussi par une mécanisation, la formalisation de la commercialisation de l'or présente des inconvénients pour les petits exploitants et les patrons. Nous avons vu plus haut que la vente de l'or est conditionnée par l'origine du financement. La vente d'une grande partie de l'or est faite auprès des patrons ou intermédiaires financiers (Mégret, 2013) qui prennent des risques pour financer les travaux. Leur exclusion de la chaîne de commercialisation de l'or contribue à la fragilisation de l'activité. Surtout que l'ANEEMAS ne propose pas de voie de financement des activités hors du circuit légal. Il est difficile pour les exploitants d'accepter de vendre leur or à la nouvelle agence suite à l'endettement perçu auprès de leurs patrons. Aussi, la vente de l'or à ces derniers à un prix supérieur à ceux de l'agence leur permet-elle de faire plus de bénéfices.

Comme le souligne Geenen (2012), les exploitants ont besoin d'incitation pour adhérer à la formalisation. Dans ce cadre, le gouvernement burkinabè a baissé les taxes d'exportation au kilogramme de l'or par le canal de l'ANEEMAS. De 450000 FCFA (environ $680 €$ ) via l'ancien canal, les taxes d'exportation du kg d'or s'élèvent à 200 000 FCFA (soit un peu plus de $300 €$ ) avec l'ANEEMAS (Medinilla et al., 2020). Les bénéfices tirés de la vente de l'or par le gouvernement ne sont ainsi pas importants, mais permettent, à travers le rapatriement des fonds dans le pays, de renforcer l'économie nationale et de limiter le blanchiment d'argent qui favorise la fraude.

Dans les sites d'extraction, le prix d'achat de l'or varie selon le statut du vendeur et les petits exploitants ne doivent pas détenir plus d'une certaine quantité d'or. Les exploitants détenteurs de la carte d'artisan minier peuvent vendre leur or à l'ANEEMAS à environ 17900 FCFA les 18 carats, 21900 les 22 et 22900 les 23 carats (il s'agit d'environ $27 €$ pour les 18 carats, $33 €$ pour les 22 et environ $35 €$ pour les 23 carats). Ces prix sont inférieurs à celui proposé par les acheteurs dans les sites de la commune de Kampti où les 22 carats peuvent atteindre $25000 \mathrm{FCFA}$ soit un peu plus de $38 €$. Ce manque à gagner explique la réticence des petits exploitants à la formalisation. 
59 La crainte des orpailleurs de la commune de Kampti se situe aussi au niveau des fouilles à la sortie des sites d'extraction en cas de formalisation des activités par le gouvernement comme au Soudan (Gagnol et al., 2019). Selon des exploitants, l'organisation et la délimitation des sites d'extraction et de traitement vont entrainer la fouille des exploitants à l'entrée et à la sortie des zones d'extraction et de traitement. Dans d'autres sites formels du Burkina Faso comme Djikando, des tickets seraient obligatoires pour l'accès aux différentes zones. Selon l'agence, l'accès sera conditionné par la détention de carte d'artisan.

\section{Conclusion}

Les analyses montrent que l'évolution des techniques d'extraction, en plus de comporter des impacts négatifs sur l'environnement, contribue à stimuler l'envie des acteurs à aller vers la formalisation. Cela, à travers le besoin de sécuriser leurs investissements comme ce fut le cas en Californie il y a plusieurs décennies (Libecap, 1986 ; Umbeck, 1977). Cependant, le penchant des textes pour les titres d'exploration et de mines industrielles ne permet pas à ces derniers d'obtenir des autorisations d'exploitation artisanale. Cela constitue un obstacle à la formalisation, comme en Colombie et en République Démocratique du Congo (Veiga et Marshall, 2018 ; Geenen, 2012). Ces contraintes juridiques ont été favorables à l'émergence d'une élite qui tire grandement profit de l'activité (Lanzano et di Balme, 2021 ; di Balme et Lanzano, 2013, 2014 ; Côte, 2013). La réorganisation formelle du milieu entamée depuis 2018 par l'ANEEMAS, se heurte à ces patrons dont la position dans la chaîne de production et de commercialisation constitue un frein à la formalisation. Cela constitue un obstacle véritable et ainsi exacerbe la fraude et l'informalité comme en Colombie (Veiga et Marshall, 2018).

61 L'exclusion pure et simple de certains acteurs clés pourrait entraîner des révoltes favorables à la clandestinité et défavorables à la formalisation durable. Pour obtenir une solution durable en matière de formalisation, il est plus que nécessaire d'intégrer, dans la mesure du possible, chaque type d'acteur de la chaîne de production et de commercialisation de l'or artisanal. Cela pourrait se faire en accompagnant certains de ces acteurs à se reconvertir à d'autres activités de la chaîne de production et de commercialisation ou à des activités indirectement liées à l'orpaillage.

L'échec de la formalisation du secteur minier artisanal montre ainsi une inefficacité de la gouvernance centralisée qui présente une préférence pour les mines formelles à grande échelle au détriment de l'exploitation artisanale de l'or. Il est alors opportun d'aller vers une gouvernance locale de l'exploitation artisanale de l'or. Cet article ouvre ainsi une réflexion sur la gouvernance locale de l'orpaillage pour une meilleure formalisation. 


\section{BIBLIOGRAPHIE}

Almaden C. R. C., 2015. Political Ecology of the Small-scale Gold Mining in Cagayan de Oro City, Philippines. Mediterranean Journal of Social Sciences, [En ligne], vol. 6, $\mathrm{n}^{\circ} 1$-S1, p. 351-362. URL: https://www.richtmann.org/journal/index.php/mjss/article/view/5547 - DOI: https://doi.org/ 10.5901/mjss.2015.v6n1s1p351

Alvarez Y. B., Coué B., Schein P. (2016). Filières de Commercialisation de l'or artisanal en Afrique de l'Ouest: Une étude de la chaîne d'approvisionnement de deux régions aurifères, au Burkina Faso et au Sénégal [En ligne]. Alliance pour une Mine Responsable, 32 p. URL: http://

www.responsiblemines.org/wp-content/uploads/2017/10/Publication-filieres-decommercialisation-or-Afrique-ouest.pdf

Balme L. A. di, Lanzano C., 2013. Entrepreneurs de la frontière » : Le rôle des comptoirs privés dans les sites d'extraction artisanale de l'or au Burkina Faso. Politique africaine, $n^{\circ} 131$, p. 27-49.

di Balme L. A., Lanzano C., 2014. Étude sur l'organisation technique et politique de deux sites d'orpaillage (Bantara et Gombélèdougou, Burkina Faso). Étude RECIT, nº37.

Bamba O., Pélédé S., Sako A., et al.., 2013. Impact de l'artisanat minier sur les sols d'un environnement agricole aménagé au Burkina Faso. Journal des Sciences, vol. 13, nº 1, p. 1-11.

Barney K., 2018. Reassembling informal gold mining for development and sustainability? Opportunities and limits to formalisation in India, Indonesia and Laos. In Lahiri-Dutt K., Between the Plough and the Pick: Informal, artisanal and Small-Scale Mining in The contemporary world. Canberra, ANU Press, p. 324-369.

Beavis S., McWilliam A., 2018. Muddy rivers and toxic flows : Risks and impacts of artisanal goldmining in the riverine catchments of Bombana, Southeast Sulawesi (Indonesia). In Lahiri-Dutt K., Between the Plough and the Pick: Informal, artisanal and Small-Scale Mining in The contemporary world. Canberra, ANU Press, p. 295-310.

Bohbot J., 2017. L'orpaillage au Burkina Faso : une aubaine économique pour les populations, aux conséquences sociales URL: http://journals.openedition.org/echogeo/15150 - DOI : https:// doi.org/10.4000/echogeo.15150

Bolay M., 2016. « Il faut être là où l'or sort! » De l'itinérance temporaire au maintien d'un mode de vie mobile chez les orpailleurs de Haute Guinée. Stichproben. Wiener Zeitschrift für kritische Afrikastudien [En ligne], vol. 16, n 30, p. 111-135. URL: https://stichproben.univie.ac.at/ fileadmin/user_upload/p_stichproben/Artikel/Nummer30/Stichproben_30_06_Bolay.pdf - DOI: https://doi.org/10.25365/phaidra.266_06

Bolay M., 2018. Reversed rushes? Expulsion as a dominant feature of gold miners' mobility in Guinea and Mali. Ressource World [En ligne]. URL: http://resourceworlds.org/2018/02/25/ reversed-rushes-expulsion-as-a-dominant-feature-of-gold-miners-mobility-in-guinea-and-mali/ Calkins S., 2016. How “Clean Gold" Game to Matter: Metal Detectors, Infrastructure, and Valuation. Hau: Journal of Ethnographic Theory, vol. 6, n 2, p. 173-195.

Caüuet B., 2000. Techniques de boisage dans les mines d'or gauloises du sud-ouest du Massif central : Mines et métallurgies en Gaule. Gallia [En ligne], tome 57, p. 129 146. DOI: https:// doi.org/10.3406/galia.2000.3214

Chevrillon-Guibert R., 2017. Le Boom de l'or au Soudan : Enjeux et perspectives pour les acteurs nationaux. International Development Policy - Revue internationale de politique de développement [En 
ligne], n 7-1. URL: http://journals.openedition.org/poldev/2231 - DOI: https://doi.org/10.4000/ poldev.2231

Chevrillon-Guibert R., Magrin G., 2018. Ruées vers l'or au Soudan, au Tchad et au Sahel : Logiques étatiques, mobilités et contrôle territorial. Bulletin de l'association de géographes français [En ligne], vol. 95, n 2, p. 272 289. URL: http://journals.openedition.org/bagf/3272 - DOI: https://doi.org/ $10.4000 /$ bagf.3272

Coavoux S., 2013. Marcel Mauss, Techniques, technologie et civilisation. Lectures [En ligne]. URL: http://journals.openedition.org/lectures/10288 - DOI : https://doi.org/10.4000/lectures.10288

Dessertine A., 2016. From pickaxes to metal detectors: Gold mining mobility and space in Upper Guinea, Guinea Conakry. The Extractive Industries and Society, vol. 3, n² 2, p. 435-441.

Fisher E. (2008). Artisanal gold mining at the margins of mineral resource governance : A case from Tanzania. Development Southern Africa, vol. 38, n 4, p. 735-760.

Gagnol L., Magrin G., Chevrillon-Guibert R., 2019. Chami, ville nouvelle et ville de l'or. Une trajectoire urbaine insolite en Mauritanie. L'Espace Politique [En ligne], $\mathrm{n}^{\circ}$ 38. URL: http:// journals.openedition.org/espacepolitique/6562 - DOI: https://doi.org/10.4000/espacepolitique. 6562

Geenen S., 2012. A dangerous bet. The challenges of formalizing artisanal mining in the Democratic Republic of Congo. Resources Policy, vol. 37, n 3, p. 322-330.

Geenen S., 2014. 'Qui cherche, trouve' the political economy of access to gold mining and trade in South Kivu, DRC. Doctorat, University of Antwerp. 364 p.

Gros M., Mégret, Q., 2018. L'or, le sang, la pluie et les génies : Chroniques ethnographiques d'un conflit entre orpailleurs et autochtones lobi du Sud-Ouest burkinabè. Afrique contemporaine [En ligne], $n^{\circ}$ 267-268, p. 113-134.

Hilson G., 2002. The environmental impact of small-scale gold mining in Ghana : Identifying problems and possible solutions. The Geographical Journal, vol. 168, n 1, p. 5772.

Human Rights Watch, 2011. Un mélange toxique Travail des enfants, mercure et orpaillage au Mali. Human Rights Watch, $132 \mathrm{p}$.

Jyotishi A., Lahiri-Dutt K., Sivramkrishna S., 2018. Historical trajectory of goldmining in the Nilgiri-Wayanad region of India. In Lahiri-Dutt K., Between the Plough and the Pick : Informal, artisanal and Small-Scale Mining in The contemporary world. Canberra, ANU Press. p. 235-259.

La Croix S. J., 1992. Property Rights and Institutional Change during Australia's Gold Rush. Explorations in Economic History, $\mathrm{n}^{\circ}$ 29, p. 204-227.

Lanzano C., di Balme L. A., 2021. Who owns the mud? Valuable leftovers, sociotechnical innovation and changing relations of production in artisanal gold mining (Burkina Faso). Journal of Agrarian Change, vol. 21, $\mathrm{n}^{\circ}$ 3, p. 433-458.

Libecap G. D., 1986. Property rights in economic history : Implications for research. Explorations in Economic History, $\mathrm{n}^{\circ}$ 23, p. 227-252.

Magrin G., 2017. Orpaillage illégal au Fitri (Tchad central). Image à la une de Géoconfluences [En ligne]. URL: http://geoconfluences.ens-lyon.fr/informations-scientifiques/a-la-une/image-a-laune/magrin-orpaillage-tchad

Maréchal L., 2013. Le secteur minier est-il porteur de développement en Afrique ? Politique étrangère, $\mathrm{n}^{\circ}$ 2, p. 85 à 98. 
Medinilla A., Karkar, P., Zongo T., 2020. Encadrer à nouveau l'artisanat minier au Burkina Faso. Vers une approche contextualisée. Ecdpm, Document de réflexion [En ligne] n² 270, 29 p. URL: https://ecdpm.org/fr/publications/encadrer-artisanat-minier-burkina-faso-approchecontextualisee/

Mégret Q., 2013. «L'argent de l'or ». Exploration anthropologique d'un « boom » aurifère dans la région Sud-Ouest du Burkina Faso. Thèse de doctorat, Université Lumière Lyon 2.

Ministère des Mines et de l'Énergie, 2013. Politique sectorielle de l'Énergie 2014-2025. Ministère des Mines et de l'Énergie. 55 p.

ONUDI., 2009. Atelier sous-régional d'information des pays de l'Afrique de l'ouest francophone sur les problèmes lies a l'orpaillage. ONUDI, $34 \mathrm{p}$.

Razanamahandry C. L., 2017. Pollution environnementale par le cyanure et potentialités de la bioremédiation dans des zones d'extraction aurifère en Afrique Subsaharienne: Cas du Burkina Faso. Thèse de Doctorat, Institut International d'Ingénierie de l'Eau et de l'Environnement.

Richard M., Moher P., Hamza D., 2015. La santé dans l'orpaillage et l'exploitation minière artisanale. Un manuel pour instructeurs. ONUDI, Artisanal Gold Council, 66 p.

Roamba J., 2014. Risques environnementaux et sanitaires sur les sites d'orpaillage au Burkina Faso: Cycle de vie des principaux polluants et perceptions des orpailleurs (cas du site Zougnazagmligne dans la commune rurale de Bouroum, région du Centre-nord). Master, Institut International d'Ingénierie de l'Eau et de l'Environnement. $101 \mathrm{p}$.

Sawadogo E., 2011. L'impact sanitaire de l'exploitation artisanale de l'or : Cas du site de Fofora dans la province du Poni. Mémoire de maîtrise, Université Pr. Joseph Ki-Zerbo. 114 p.

Sawadogo E., 2021. Discours, pratiques et dynamiques environnementales autour de l'orpaillage dans la commune de Kampti (Sud-Ouest du Burkina Faso). Thèse de doctorat, Université Paris 1 Panthéon Sorbonne, Paris et Université Joseph Ki-Zerbo, Ouadougou. 342 p.

Sawadogo E., Da D. E. C., 2020. Orpaillage et dynamiques des modes d'accès aux ressources naturelles à Kampti. Revue des Lettres et Sciences Sociales du PASRESS, vol. 8, n² 29, p. 106-125.

Siegel S., Veiga M. M., 2009. Artisanal andsmall-scale mining as an extralegal economy :De Soto and the redefinition of "formalization". Resources Policy, n" 34, p. 5156.

Tschakert P., Singha K., 2007. Contaminated identities : Mercury and marginalization in Ghana's artisanal mining sector. Geoforum, $\mathrm{n}^{\circ} 38$, p. 1304-1321.

Umbeck J., 1977. The California Gold Rush : A Study of Emerging Property Rights. Explorations in Economic History, nº 14, p. 197-226.

UNITAR et ONU Environnement., 2018. Manuel pour développer des stratégies de formalisation de l'EMAPE d'or, dans le cadre des Plans d'Action Nationaux. UNITAR et ONU Environnement, $134 \mathrm{p}$.

Veiga M. M., Marshall, B. G., 2018. The Colombian artisanal mining sector: Formalization is a heavy burden. The Extractive Industries and Society, année 2019.

Werthmann K., 2003. "Ils sont venus comme une nuée de sauterelles" Chercheurs d'or au Burkina Faso. In Kuba R., Lentz C., Somda C. N. (ed.), Histoire du peuplement et relations interethniques au Burkina Faso. Karthala et ZMO, p. 97-110.

World Bank, 2005. On a credit in the amount of us $\$ 20.14$ million to Burkina Faso for a mining sector capacity building and environmental management project. Word Bank, $\mathrm{n}^{\circ} 31518,50 \mathrm{p}$. 


\section{NOTES}

1. L'« informalité » est le déroulement d'une activité hors des circuits formels établis par l'État. Cela rend difficile son contrôle par les institutions formelles entraînant sa faible contribution à l'économie formelle.

2. Selon le code minier de 2015, l'autorisation d'exploitation artisanale de substances de mines est accordée, sous réserve des droits antérieurs, par décision de l'Administration des mines, après avis du ministre chargé de l'environnement, des autorités administratives compétentes et des collectivités territoriales concernées aux personnes physiques burkinabè ou aux ressortissants des pays accordant la réciprocité aux burkinabè et aux coopératives intervenant dans le secteur minier.

3. URL: http://lesaffairesbf.com/2014/07/20/vente-de-detecteurs-dor-une-bonne-affaire-agaoua/. Consulté le 29 juillet 2020

4. 2001 correspond à une période de quasi-absence de l'exploitation artisanale de l'or dans la commune.

5. URL: pasmep.org/.../58-kampti-mort-de-huit-boeufs-probablement-intoxiques-aucyanure. Consulté le 16 juillet 2021.

6. La norme de concentration maximale des eaux de boissons en mercure inorganique est $0,001 \mathrm{mg} / \mathrm{l}$ selon l'OMS.

7. Les 30 et 31 octobre 2014, une insurrection populaire a eu lieu sur presque toute l'étendue du territoire pour dire non à la modification de l'article 37 de la constitution qui avait pour but de permettre au chef d'État Blaise Compaoré au pouvoir depuis 1984 de se représenter.

8. Décret $\mathrm{n}^{\circ}$ 2018-1017 PRES/PM/MMC/MINEFID/. MEEVCC/MCIA/MATD/MSECU/MFPTPS portant organisation des exploitations artisanales et semi-mécanisées de l'or et autres substances précieuses.

\section{RÉSUMÉS}

La formalisation de l'exploitation artisanale de l'or est recommandée par la Banque mondiale qui considère qu'elle favorise la minimisation des impacts néfastes. Selon certains auteurs, la mécanisation et le niveau élevé du prix de l'or à l'échelle internationale sont favorables à une formalisation efficace. Sur la base de recherches documentaires et d'enquêtes de terrain dans la commune de Kampti en mai 2018 et décembre 2019, nous démontrons que malgré la volonté des acteurs à sécuriser leurs investissements par la formalisation, ils se heurtent à des réalités institutionnelles et juridiques défavorables. Dans la nouvelle perspective de formaliser les sites d'or par l'Agence nationale d'encadrement des exploitations minières artisanales et semimécanisées (ANEEMAS), la mise à l'écart d'acteurs clés de la chaîne de commercialisation limite l'efficacité du processus de formalisation de l'activité.

The formalization of artisanal gold mining is recommended by the World Bank, which considers that it promotes the minimization of harmful impacts. According to some authors, mechanization and the high level of the price of gold internationally are favorable to effective formalization. On the basis of documentary research and field surveys in the commune of Kampti in May 2018 and December 2019, we show that despite the willingness of actors to secure their investments through formalization, they come up against unfavorable institutional and legal 
realities. In the new perspective of formalizing gold sites by National Agency for the Supervision of Artisanal and semi-mechanized mining (ANEEMAS), the sidelining of key players in the marketing chain limits the effectiveness of the process of formalizing the activity.

INDEX

Thèmes : Sur le Champ

Keywords : mining technique, gold, formalization, Kampti, Burkina Faso

Mots-clés : technique d'exploitation, or, formalisation, Kampti, Burkina Faso

\section{AUTEURS}

EDITH SAWADOGO

Edith Sawadogo, editsawadogo@yahoo.fr ou editsabarry@gmail.com est docteur en géographie. Elle a récemment publié :

- Sawadogo E., Da Dapola E. C., 2020. Orpaillage et dynamiques des modes d'accès aux ressources naturelles à Kampti. Revue des Lettres et Sciences Sociales du PASRESS, vol. 8, n² 29, p. 106-125. 